\title{
Electrocutaneous stimulation III. The perception of temporal order
}

\author{
LAWRENCE E. MARKS \\ John B. Pierce Foundation Laboratory and Yale University, New Haven, Connecticut \\ JOHN P. GIRVIN \\ The University of Western Ontario, London, Ontario, Canada \\ and \\ MICHAEL D. O'KEEFE, PETER NING, DONALD O. QUEST, \\ JOHN L. ANTUNES, and Wm. H. DOBELLE \\ College of Physicians and Surgeons of Columbia University, New York, New York
}

\begin{abstract}
Temporal order thresholds were measured for brief electrical stimuli presented to the forehead and to the abdomen. Temporal acuity is better (thresholds smaller) on the forehead, sensitivity increasing somewhat (thresholds decreasing) as the phyeical separation between the electrodes increases. Even in that subject, of four, who had the lowest thresholds, however, the value rarely fell below $100 \mathrm{msec}$. Temporal order thresholds also depend on the orientation, being far smaller when the electrodes are separated horizontally than when separated vertically on both body sites.
\end{abstract}

The first paper in this series examined several parameters affecting absolute sensitivity to electrocutaneous stimulation (Girvin, Marks, Antunes, Quest, O'Keefe, Ning, \& Dobelle, 1982). The second assessed the skin's ability to represent spatial information (Marks, Girvin, Quest, Antunes, Ning, O'Keefe, \& Dobelle, 1982). The aim of this third paper is to evaluate the skin's capacity to process temporal information: any sensory substitution device will perforce have to operate in time; the interpretation of information presented electrically to the skin will rest upon discriminations and identifications of temporally dynamic spatial displays.

A fundamental constraint on the informationprocessing capacity of any sensory system comes from its limited ability to make discriminations in the time domain. One measure of this limitation is the temporal order threshold-the maximal rate at which a person can correctly identify the order of successive stimuli. Classical measurements by Hirsh and Sherrick (1961) found the temporal order threshold to be about $20 \mathrm{msec}$ in several sense departments (touch, vision, hearing) and under a variety of conditions, although subsequent research (e.g., Westheimer \& McKee, 1977) suggests that

This research was supported by a grant from the National Science Foundation (PFR 79-17634). Reprints may be requested from Lawrence E. Marks, John B. Pierce Foundation Laboratory, 290 Congress Avenue, New Haven, Connecticut 06519. D. O. Quest and J. L. Antunes are affiliated with the Neurological Institute of New York, and M. D. O'Keefe, P. Ning, and W. H. Dobelle are affiliated with the Division of Artificial Organs, both of the College of Physicians and Surgeons of Columbia University. the exact value of an order threshold can depend on the exact stimulus parameters used. With the long-range goal of developing an electrocutaneous stimulator capable of providing speech information to the deaf, the present study examined temporal order thresholds for electrical stimulation.

As just mentioned, Hirsh and Sherrick (1961) measured temporal order thresholds in touch, vision, and hearing. Under a variety of stimulus conditions, they found the order threshold to be virtually invariantequal to about $20 \mathrm{msec}$ on the average-in well-practiced subjects. In other words, $20 \mathrm{msec}$ between the onsets of two stimuli sufficed for subjects to identify, on $75 \%$ of the trials, which of the two came first. In fact, Hirsh and Sherrick found the same threshold value of $20 \mathrm{msec}$ even when one stimulus was presented to one modality, the other to a different modality. This finding suggests that the order threshold may reflect limited temporal resolving capacity at a central location-a location that processes multimodal inputs-rather than limited temporal resolving capacity within a single modality.

Indeed, thresholds of $20.30 \mathrm{msec}$, or even the $3 \mathrm{msec}$ reported by Westheimer and McKee (1977) for perceived visual order under appropriate spatial conditions, may not mean that the ultimate limit on temporal resolution is $1 / 50$ th or even $1 / 300$ th of a second. It is well known that two auditory pulses whose onsets are separated by less than $1 \mathrm{msec}$ can be discriminated, in that pulse $A$ to one ear followed by pulse B to the other differs perceptually from the reverse sequence, $B$ followed by $A$. This happens because the two sequences are lateralized differently: the person hears one sound, and it is heard at the 
ear that received the first pulse. The significant fact here is that what is heard is a single sound. By contrast, to ask about temporal order thresholds is to ask, What is the time interval needed between stimulus onsets to identify their order, given that the two stimuli do not fuse perceptually but maintain their identities?

This study asks about temporal order sensitivity to two stimuli separated spatially on the skin. Measurements like these have been reported by Hirsh and Sherrick (1961), by Saunders (1974), and by Sparks (1979), who assessed the minimal time needed for subjects to determine the spatial direction of sequentially activated electrodes; under certain conditions, Sparks found temporal thresholds to be as small as $5 \mathrm{msec}$.

The present study seeks answers to five main questions: (1) In general, how "good" is the skin at making temporal order discriminations with electrical stimuli? (2) How do temporal order thresholds depend on locus of stimulation on the skin? (3) How do temporal order thresholds depend on the physical distance between electrodes? (4) How do temporal order thresholds depend on orientation of the two electrodes? (5) How much do temporal order thresholds vary across individuals?

\section{METHOD}

\section{Stimuli}

The stimuli were constant-current, biphasic $(+/-)$ pulses of $.5 \times .5 \mathrm{msec}$ duration, delivered through concentric electrodes previously described (Girvin et al., 1982). Each stimulation comprised a pair of biphasic pulses, the time interval between the onset of the first biphasic pulse and the onset of the second pulse-the stimulus onset asynchrony, or SOA-determined by the computer.

\section{Procedure}

The subject's skin was moistened, and the electrodes were attached and held in position by tape and an elastic bandage. In the main experiments, four electrodes were placed on the skin of the forehead or abdomen, spaced horizontally to provide distances between various pairs of electrodes, center to center, of $40,70,100$, and $140 \mathrm{~mm}$.

The subject sat in a comfortable chair, located in a soundattenuated booth. The subject responded to stimulation by pressing appropriate buttons on a touchtone keyboard, whose output fed to the computer. Audible signals presented through a speaker in the booth coincided with electrical stimulation.

First, absolute thresholds were measured for each electrode as before (Girvin et al., 1982). For the temporal order measurements, stimulus levels were set at 2.5 times threshold, with the restriction that the greatest current available was $8 \mathrm{~mA}$.

In measuring temporal order thresholds, two procedures were tested. The main procedure was as follows. Two electrodes were selected to give the desired distance, and the subject was given the opportunity to present each of the stimuli to himself, so as to identify one as A, the other as B. Once the subject was ready, he could proceed with the threshold measurement. The first pair of stimuli had a stimulus onset asynchrony (SOA) of $2 \mathrm{sec}$ (the greatest value available); the order, $\mathrm{AB}$ or $\mathrm{BA}$, was randomly determined, and the subject had to decide which order was presented. Only after correct responses to three consecutive stimulations was the SOA decreased on the following trial by half, whereas one incorrect response caused the SOA to double on the following trial. Once the lower limit was established by the first "no" and the upper limit by the first subsequent "yes" (by same rule of three correct before a decrease, one incorrect before an increase), each succeeding stimulus was presented at a duration halfway between that of the previous stimulus and the limit being approached; each reversal set a new limit. Thus, the stimuli bracketed threshold in smaller and smaller steps, and the run terminated when the change in duration was $10 \%$ of the previous stimulus. This stimulus was defined as threshold for the run. As a control for this procedure, some thresholds were also measured using a standard forced-choice staircase procedure in which the stimulus duration increased (after one incorrect response) or decreased (after three consecutive correct responses) by a factor of $25 \%$. This staircase procedure continued until 12 reversals of direction occurred. The durations of the last 10 turnaround points were averaged to give the temporal order threshold. Under both procedures, the threshold corresponds to the value correctly discriminated $80 \%$ of the time (Wetherill \& Levitt, 1965). The second procedure was tested on two subjects in the conditions of the main experiment described below, and found to produce substantially the same outcome as the first procedure. (A similar outcome obtained in pilot research in which the threshold was approached from below rather than above.) Because the first procedure was much more rapid, it was used throughout.

The main experiment investigated temporal order sensitivity on the forehead and on the abdomen, with horizontal separations between electrodes of $40,70,100$, and $140 \mathrm{~mm}$. In this experiment, each subject served in two sessions using each locus, making four sessions per subject in all. In each session, five threshold measurements were made for each distance-these being done in random order; thus, 10 measurements in all were made for each locus and distance for each subject.

$\dot{A}$ secondary experiment investigated thresholds on the forehead and abdomen with constant separations of $40 \mathrm{~mm}$, but with vertical as well as horizontal orientations of electrodes. Here, each subject served in two sessions, one for each locus; in each session, 10 thresholds were measured for each orientation.

Four men, including two of the authors, served as subjects in both experiments.

\section{Results}

Figures 1 and 2 show, for the forehead and abdomen, respectively, the median temporal order threshold for

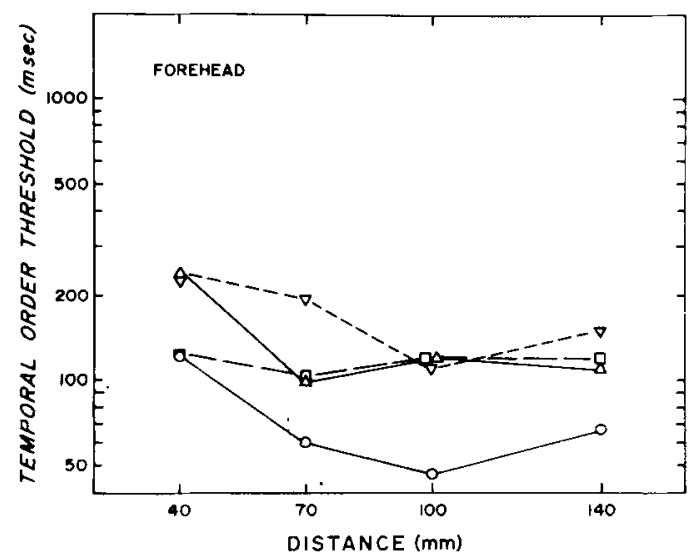

Figure 1. Temporal order thresholds in milliseconds as a function of the distance separating electrocutaneous stimuli on the forehead for four subjects. 


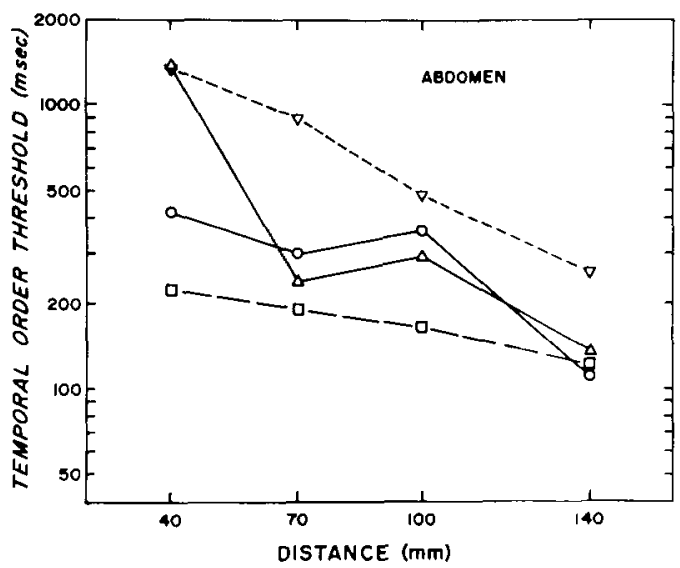

Figure 2. As in Figure 1, for the abdomen.

each subject, plotted as a function of the distance between the electrodes. (Medians were computed because, on some runs, threshold values were greater than $2,000 \mathrm{msec}$ and therefore were not measurable.) Four features distinguish themselves.

(1) Temporal order sensitivity is uniformly poor for these electrocutaneous stimuli. Most of the threshold values for the forehead and all of the values for the abdomen are greater than $100 \mathrm{msec}$-under some conditions and for some subjects greater than $1 \mathrm{sec}$ ! These values are considerably greater than the value of $20 \mathrm{msec}$ reported by Hirsh and Sherrick (1961) for vibratory touch and for vision and hearing.

(2) Sensitivity is greater on the forehead than on the abdomen. At the shorter stimulus distances-40 and $70 \mathrm{~mm}$-the temporal order thresholds are 2.3 times higher on the abdomen, although at the greatest dis-

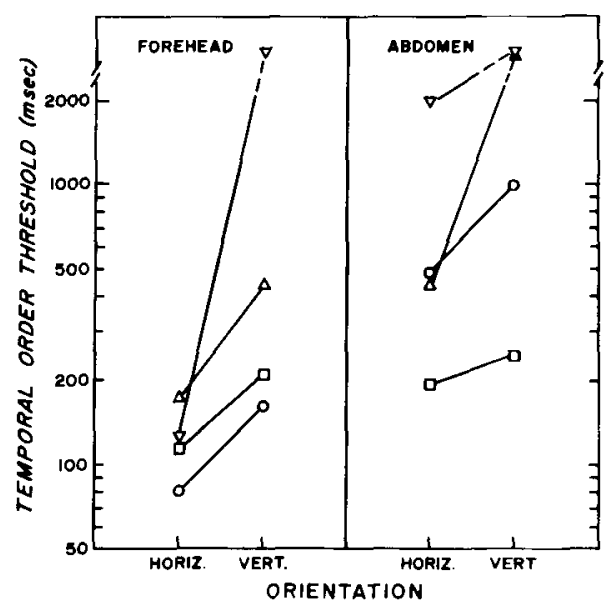

Figure 3. Temporal order thresholds in milliseconds for horizontal and vertical orientations of electrocu taneous stimuli on the forehead and abdomen, for four subjects. tance-140 mm-thresholds are more similar on the two body sites.

(3) Notably on the abdomen, but on the forehead too, temporal order thresholds decline as distance between electrodes increases. In part or in whole, this may represent an improvement closely tied to spatial acuity: on the abdomen in particular, and for two of the four subjects especially, the stimuli when separated by only $40 \mathrm{~mm}$ were difficult to distinguish spatially.

(4) Individual differences are clear. L.M. gave thresholds on the forehead that generally fell below $100 \mathrm{msec}$, while the other three subjects gave results similar to one another and higher in value. On the abdomen, J.G. clearly was the most sensitive subject and H.H. was the least sensitive.

Figure 3 compares median thresholds to stimuli spaced horizontally and vertically on both the forehead and the abdomen, thereby making a fifth main point:

(5) Orientation clearly makes an enormous difference to temporal order sensitivity, with the horizontal orientation yielding much greater temporal resolution (lower thresholds). Indeed, for one subject on the forehead and for two subjects on the abdomen, the median threshold for the vertical orientation could not be measured, being greater than the 2-sec maximum SOA that was available. Again, under nearly all conditions for all subjects, temporal order thresholds are relatively high in value, running, with one exception, between 80 and $450 \mathrm{msec}$ on the forehead and, with two exceptions, between 200 and $2,000 \mathrm{msec}$ on the abdomen.

\section{DISCUSSION}

The findings of this study have two implications, one theoretical and the other practical. The theoretical implication is that the skin has poor temporal resolving capacity when stimulated electrically, at least under the present conditions. Recall the conclusion that can be drawn from Hirsh and Sherrick's (1961) study, namely that their threshold value of $20 \mathrm{msec}$ may represent a limit imposed by a central processing system that monitors information coming from several individual modalities. The individual modalities themselves-vibratory touch, vision, hearing-may have even greater temporal resolving power, but cannot display it because of the limitation of the central monitor. By way of contrast, the considerably higher threshold values measured here (to the extent they are not based on procedural differences-see below) presumably arise not from any limitation imposed by such a putative central mechanism, but rather from some limitation on the temporal resolving power of the cutaneous system that derives from the use of electrical stimuli.

This conclusion must be evaluated in the light of the results reported by Saunders (1974), Sherrick (1970), and Sparks (1979). Sherrick (1970), using electrodes separated by $90 \mathrm{~mm}$ transversely on the thigh, measured 
a temporal order threshold of $36 \mathrm{msec}$; Saunders (1974), using the abdomen, mentions measuring a threshold of $40 \mathrm{msec}$. One possible reason for these lower values is procedure. Sherrick (1970) followed the method of Hirsh and Sherrick (1961), which allowed multiple presentations of each stimulus pattern before judgment (Gengel \& Hirsh, 1970). By contrast, the present experiments employed single presentations, a method known to yield longer temporal order thresholds (see Gengel $\&$ Hirsh, 1970). The single-presentation procedure was selected because it more closely simulates the decision situation expected of a person using a real-time, electrocutaneous communication device. Such situations can be far from ideal with respect to temporal order discriminability.

Sparks (1979) reported that under certain circumstances the temporal threshold for determining the direction of activation of a linear array of electrodes could be as small as $5 \mathrm{msec}$. It is important to note the many differences-both in stimulus arrangement and in procedure-between Sparks's study and the present one. First of all, Sparks's thresholds were smallest on the forearm (that is, smaller on the forearm than on the abdomen, these being the two body sites examined). Moreover, the smallest threshold $(5 \mathrm{msec})$ obtained when eight electrodes were activated in sequence on the forearm. When eight electrodes were activated in sequence on the abdomen, the threshold rose to $14 \mathrm{msec}$.

Perhaps most comparable to conditions in the present experiment was the condition in Sparks's (1979) study (his Experiment 2) in which "two" electrodes separated horizontally by about $180 \mathrm{~mm}$ were activated on the abdomen; that condition gave a threshold of $58 \mathrm{msec}$. Linear extrapolation of the data in the present study (Figure 2) to a separation of $180 \mathrm{~mm}$ on the abdomen yields an estimated average temporal order threshold of about $100 \mathrm{msec}$. Note, however, that in Sparks's study each stimulus electrode was actually a vertical column of four electrodes; on the abdomen, Sparks found the temporal threshold for a single electrode (his Experiment 1) to be about twice that for a column of four. It may be, therefore, that under strictly comparable conditions the results of Sparks's study and of the present one would be in good agreement.

Other differences exist between the studies. Sparks (1979). employed bursts of pulses, each pulse within a burst lasting only $5-25 \mathrm{msec}$. Another difference is in procedure: Sparks used a two-alternative forced-choice method. On each trial, the subject was given two intervals, one with the electrodes activated in one direction and the other with the electrodes activated in the opposite direction. The task was to identify which interval contained the stimulus that went from the midline (abdomen) or from the elbow (forearm). Feedback was given after each trial. It is conceivable that this procedure might enable the subjects to pick up any possible differ- ence between stimuli, and thus to yield relatively low thresholds (although informal experiments that we have conducted suggest feedback not to have any substantial effect).

Given the differences in stimulus conditions and procedure, the seemingly great discrepancy between temporal thresholds reported here and those reported by Sparks appears less remarkable. There is, however, one significant difference between findings in the two studies, namely with respect to the effect of orientation of the stimuli. As Figure 3 shows, temporal order thresholds are far lower with horizontal than with vertical separations between electrodes. Sparks (1979) reports data obtained with horizontal separations, but states that vertical separations gave nearly identical results. We see no obvious explanation for the discrepancy on this point.

The main theoretical implication of the present study is that, given activation of only two electrodes, temporal order sensitivity to electrocutaneous stimulation can be relatively poor. This theoretical implication bears on the practical implication, namely on the development of an electrocutaneous device to transmit speech information. To the extent that rapid spatiotemporal order judgments may be needed, the results warn against vertical spacing of electrodes on the skin; and to the extent that certain judgments must be based on activation of only two electrodes, the results suggest the utility of relatively wide separations between them-separations of $100 \mathrm{~mm}$ and more.

The practical implications of the present experiment bear on the design of an electrocutaneous speech aid. If such a device were to use a linear array of electrodes, the present results suggest the utility of relatively wide spacing (100 $\mathrm{mm}$ or more) in the horizontal direction (vertical avoided), at least on the forehead and abdomen. Even so, if temporal order discrimination does set an ultimate limit on perceptual processing through such a device, the present outcome implies that information transmission may be constrained to rates not much greater than one item per 100 msec.

\section{RDTERENCES}

Gencet, R. W., \& Hrkst, I. J. Temporal order: The effect of single versus repeated presentations, practice, and verbal feedback. Perception \& Psychophysics, 1970, 7, 209-211.

Girvin, J. P., Manks, L. E., Antunes, J. L., Quegt, D. O., Ning, P., O'Kese, M. D., \& DoBelle, W. D. Electrocutaneous stimulation $I$. The effects of stimulus parameters on absolute threshold. Perception \& Psychophysics, 1982, 32, 524528 .

Hinar, I. J., \& Shennick, C. E., Ja. Perceived order in different sense modalities. Journal of Experimental Psychology, $1961,62,423-432$.

Mark8, L. E., Ginvin, J. P., Quest, D. O., Antunes, J. L., Nina, P., O'KeEre, M. D., \& DobeLLe, W. H. Electrocutaneous stimulation II. The estimation of distance between two points. Perception \& Psychophysics, 1982, 32, 529-536. 
SAundens, F. A. Electrocutaneous displays. In F. A. Geldard (Ed.), Cutaneous communication systems and devices. Austin, Tex: Psychonomic Society, 1974.

Sherrick, C. E. Temporal ordering of events in haptic space. IEEE Transactions on Man-Machine Systems, 1970, MM5-11, 25-28.

Spark8, D. W. The identification of the direction of electrocutaneous stimulation along lineal multistimulator arrays. Perception \& Psychophysics, 1979, 25, 80-87.
Wegtheimer, G., \& McKex, S. P. Perception of temporal order in adjacent visual stimuli. Vision Research, 1977, 17, 887-892.

WeTHerILL, G. B., \& LevitT, H. Sequential estimation of points on a psychometric function. British Journal of Mathematical and Statistical Psychology, 1965, 18, 1-10.

(Manuscript received January 4, 1982;

revision accepted for publication September $24,1982$. 